\title{
Endoscopic ultrasound-guided transmural drainage of post-traumatic pancreatic fluid collections
}

\author{
Surinder Singh Ranaa, Ravi Sharmaa, Lovneet Dhalariaa, Rajesh Guptab \\ Postgraduate Institute of Medical Education and Research (PGIMER), Chandigarh, India
}

\section{Abstract}

\begin{abstract}
Background Pancreatic injury is an uncommon consequence of abdominal trauma, and surgery has been the conventional treatment. The role and timing of endoscopic ultrasound (EUS)-guided treatment of the consequences of traumatic pancreatic injury is unclear. Our study evaluated the safety and efficacy of EUS-guided transmural drainage of post-traumatic pancreatic fluid collections (PFC).

Methods A retrospective analysis of 13 patients (mean age $20.2 \pm 4.4$ years; 12 males) with posttraumatic PFC treated with EUS-guided transmural drainage over the last 10 years was performed. Patient demographics, imaging findings, size of PFC, details of endoscopic transmural drainage procedure, outcome details, as well complications were retrieved from our database.

Results The patients underwent drainage at $26.8 \pm 7.4$ days after abdominal trauma, and the mean size of PFC was $11.8 \pm 3.2 \mathrm{~cm}$ with 2 patients having multiple fluid collections. Ten patients had PFC with a well-formed wall and 3 patients had an incompletely formed wall. Endoscopic drainage was technically successful in all 13 patients and 11 patients underwent transmural drainage with multiple plastic stents whereas 2 patients were treated with lumen apposing metal stents. The PFC resolved in all patients over a mean period of $2.7 \pm 0.4$ weeks. One patient developed gastrointestinal bleeding 6 days after the procedure, successfully treated with angio-embolization.
\end{abstract}

Conclusion EUS-guided transmural drainage of post-traumatic PFC is safe and effective and can be safely performed at an early phase ( $<4$ weeks) after pancreatic trauma.

Keywords Trauma, pancreatitis, endosonography, computed tomography, ascites

\section{Introduction}

Pancreatic injury is an uncommon consequence of both blunt as well as penetrating abdominal trauma [1]. Despite being uncommon, pancreatic injury is associated with a significant morbidity and mortality [1-4]. The integrity of the main pancreatic duct (MPD) is one of the most important determinants of outcome after post-traumatic pancreatic injury. Patients with intact MPD usually respond well to

Department of a Gastroenterology (Surinder Singh Rana, Ravi Sharma, Lovneet Dhalaria); bSurgical Gastroenterology (Rajesh Gupta), Postgraduate Institute of Medical Education and Research (PGIMER), Chandigarh, India

\section{Conflict of Interest: None}

Correspondence to: Prof. Surinder S Rana, MD, DM, FASGE, Department of Gastroenterology, Postgraduate Institute of Medical Education and Research (PGIMER), Chandigarh 160012, India, e-mail: drsurinderrana@gmail.com

Received 28 October 2020; accepted 22 December 2020; published online 26 February 2021

DOI: https://doi.org/10.20524/aog.2021.0607 conservative treatment whereas patients with duct disruption usually require minimally invasive or surgical intervention for a successful outcome [1-8]. Patients with MPD disruption usually present with local complications like acute pancreatitis, pancreatic fluid collections, and pancreatic fistulas [7-9].

The management of post-traumatic injury depends on the duct integrity as well as the clinical stability of the patient, with surgery being the conventional treatment. Patients with hemodynamic instability are usually operated immediately whereas stable patients are initially managed conservatively. The clinical factors that determine the timing and type of surgery include site and extent of MPD disruption, stability of the patient, and extent of another organ damage $[1,4,10,11]$. The surgical intervention involves either drainage or resection depending on the extent of pancreatic parenchymal damage $[4,11,12]$. The advent of minimally invasive endoscopic therapy has opened up an effective and minimally invasive therapeutic option for the management of patients with posttraumatic pancreatic injury. Studies evaluating endoscopic transpapillary drainage by a bridging endoprosthesis across the disrupted MPD have reported encouraging results in patients with pancreatic trauma $[4,8-10]$. However, the majority of patients with post-traumatic pancreatic injury have complete 
MPD disruption, and endoscopic transpapillary drainage in such settings is usually ineffective [13]. Moreover, endoscopic transpapillary drainage alone in patients with pancreatic fluid collections (PFC) $>6 \mathrm{~cm}$ in size is associated with an increased risk of infection as well as inadequate drainage $[1,13]$. Patients with large post-traumatic PFC can be treated with imageguided percutaneous drainage (PCD), but, in presence of complete duct disruption, PCD is associated with an increased risk of external pancreatic fistula formation [13].

Endoscopic ultrasound (EUS)-guided transmural drainage is a safe and effective minimally invasive treatment option for pseudocysts and walled off necrosis [14,15]. Also, it is safe and effective in patients with PFC associated with complete MPD disruption with the strategy of maintaining the patency of iatrogenic fistula by leaving transmural stents in situ for an indefinite period, associated with an excellent long-term outcome [16]. However, experience with EUSguided transmural drainage of post-traumatic PFC is limited to case reports $[5,17,18]$. Moreover, the timing of EUS-guided intervention is unclear and it is usually recommended to wait for at least 4 weeks for the PFC to get walled off before embarking upon EUS-guided transmural drainage [14]. In this retrospective study, we report our experience with EUS-guided transmural drainage of symptomatic post-traumatic PFC.

\section{Patients and methods}

A retrospective analysis of patients with post-traumatic PFC treated with EUS-guided transmural drainage in a pancreatology unit at a tertiary care center in North India over the last 10 years was performed. Informed consent was obtained from all the patients prior to the EUS-guided drainage procedure. Patients with severe coagulopathy, thrombocytopenia or cardio-respiratory illness precluding safe endoscopic drainage were excluded from the study. The collections were drained if patient had persistent sepsis, worsening or new onset organ failure, persistent abdominal pain, or persistent symptoms due to biliary or gastric outlet obstruction. Patient demographics, imaging findings, size of PFC, details of endoscopic transmural drainage procedure, outcome details, as well complications were retrieved from our database.

\section{EUS-guided transmural drainage}

All EUS-guided drainage procedures were performed by a single experienced endosonographist under conscious sedation using a linear scanning echoendoscope (EG-3870 UTK linear echoendoscope, Pentax Inc, Tokyo, Japan or UCT180 linear echoendoscope, Olympus Optical Co. Ltd., Tokyo, Japan). Intravenous ciprofloxacin was administered for antibiotic prophylaxis and was continued orally for 7 days. On EUS, the PFC was carefully evaluated especially for presence of solid necrotic debris as well as any abnormal surrounding blood vessel. Using an approximate visual judgment of the endoscopist, the amount of solid necrotic debris was estimated as percentage of size of the PFC. Thereafter, EUS-guided transmuraldrainage was performed under EUS and fluoroscopic guidance using a standard technique described previously [19]. Briefly, the PFC was punctured with a 19-G needle (Echotip; Cook Endoscopy, Winston-Salem, NC, USA) followed by aspiration of the contents to confirm the position of the needle. This was followed by coiling of a $0.035 / 0.025$-inch guidewire into the PFC and the transmural tract was dilated using either an endoscopic retrograde cholangiopancreatography (ERCP) cannula or a 4-mm biliary balloon dilator (Hurricane biliary balloon catheter; Boston Scientific, Natick, MA, USA or a 6-Fr cystotome. Either multiple plastic stents (1-3 in number, 7 or $10 \mathrm{Fr} ; 5 \mathrm{~cm}$ in length) or a lumen apposing metal stents (LAMS) (Nagi stent (14 or $16 \mathrm{~mm}$ ), Taewoong Medical Co., Ltd., Seoul, Korea or Plumber Stent (16 mm in diameter), MI Tech Gyeonggi-Do, 17706, Korea) were used as per the patient's preference depending upon affordability due to economic considerations and availability of health insurance. The LAMS was placed without further dilatation of the transmural tract whereas the transmural tract was further dilated up to 12-15 $\mathrm{mm}$ with a wire-guided hydrostatic balloon (controlled radial expansion-balloon; Boston Scientific, Natick, MA, USA) in patients in whom plastic stents were placed.

\section{Post-procedure care}

After the EUS-guided drainage procedure, patients were admitted and kept under observation for 48-72 h. Subsequently, depending upon the clinical situation and other injuries, the patients were discharged and followed up in the outpatient department until complete resolution of the PFC. The patients were followed up in the outpatient clinic at 2 weekly intervals for clinical evaluation as well as transabdominal ultrasound. Patients with complete clinical recovery along with resolution of PFC on ultrasound underwent computed tomography (CT) of the abdomen to confirm the resolution. Thereafter, the patients underwent ERCP to delineate the MPD anatomy. In patients with normal MPD with no disruption, the transmural stents were removed. Patients with partial duct disruption underwent placement of a transpapillary bridging stent followed by repeat ERCP at 4 weeks to document healing of duct disruption followed by removal of both the transpapillary and transmural stents. In patients with disconnected pancreatic duct (DPD), one or more transmural plastic stents were left indefinitely. Patients in whom a LAMS was placed initially, an attempt was made to replace it with one or more indefinitely placed pigtail plastic stents.

\section{Definitions}

Treatment success: Resolution of symptoms with resolution of PFC on cross-sectional imaging with no need for rescue surgery.

Complications of the procedure: The complications were diagnosed according to the American Society of Gastrointestinal Endoscopy lexicon [20]. 


\section{Results}

During the study period 13 patients (mean age 20.2 \pm 4.4 years; 12 males) with post-traumatic PFC underwent EUSguided transmural drainage (Table 1). All studied patients were symptomatic with abdominal pain and underwent EUS-guided drainage at $26.8 \pm 7.4$ days after abdominal trauma. Two patients had acute lung injury and none of the patients had any other organ injury. All patients had pancreatic injury due to blunt trauma. The mean size of PFC was $11.8 \pm 3.2 \mathrm{~cm}$ with 2 patients having multiple fluid collections. Three (23\%) patients had co-existent ascites and $2(15 \%)$ patients had left-sided pleural effusion detected on CT. Twelve (92\%) patients had less than $10 \%$ solid debris and 1 patient had $30 \%$ solid necrotic debris as evaluated on EUS. None of the patients had arterial pseudo-aneurysm or abnormal vessels in the transmural tract. Ten patients had PFC with a well-formed wall and 3 patients had an incompletely formed wall.

Endoscopic drainage was technically successful in all 13 patients and 11 patients underwent transmural drainage with multiple plastic stents whereas 2 patients were treated with LAMS (Figs. 1,2). Of 11 patients treated with plastic stents, 2 patients underwent transmural drainage with a single 7 -Fr stent, 3 patients with 2 7-Fr stents, and 6 patients with a combination of 7 - and 10-Fr stent. None of the patients required either direct endoscopic necrosectomy or further sessions of endoscopic drainage. Two patients with multiple fluid collections underwent PCD along with the endoscopic drainage. The PFC resolved in all the patients over a mean time of $2.7 \pm 0.4$ weeks.

Following endoscopic drainage, 1 patient (treated with plastic stents) developed gastrointestinal bleeding 6 days after the procedure and presented with hematemesis and melena associated with postural symptoms and tachycardia. CT angiography revealed a leaking pseudoaneurysm from the gastroduodenal artery and the patient underwent successful angioembolization. No other significant complication of the EUS-guided drainage procedure was encountered.

Following resolution of PFC, ERCP was done in all 13 patients and revealed complete MPD disruption in 11 patients (neck in 8 patients and body in 3 patients) in whom one or more transmural stents were left indefinitely. Two patients had partial duct disruption, in whom a transpapillary bridging stent ( $5 \mathrm{Fr}$ ) was placed, 4-6 weeks later a repeat ERCP was done to document healing of MPD disruption, and, following that, both the transpapillary and transmural stents were removed. These successfully treated patients have been asymptomatic over a follow-up period of $26.4 \pm 20.4$ months. Two patients with permanent indwelling transmural stent had an asymptomatic spontaneous migration of transmural stents and both these patients have been asymptomatic until the last follow up.

\section{Discussion}

Traumatic injury to pancreas, although uncommon, is associated with significant morbidity because of consequences of disruption of the MPD. The disruption of MPD leads to continuous leakage of pancreatic juices with digestive enzymes

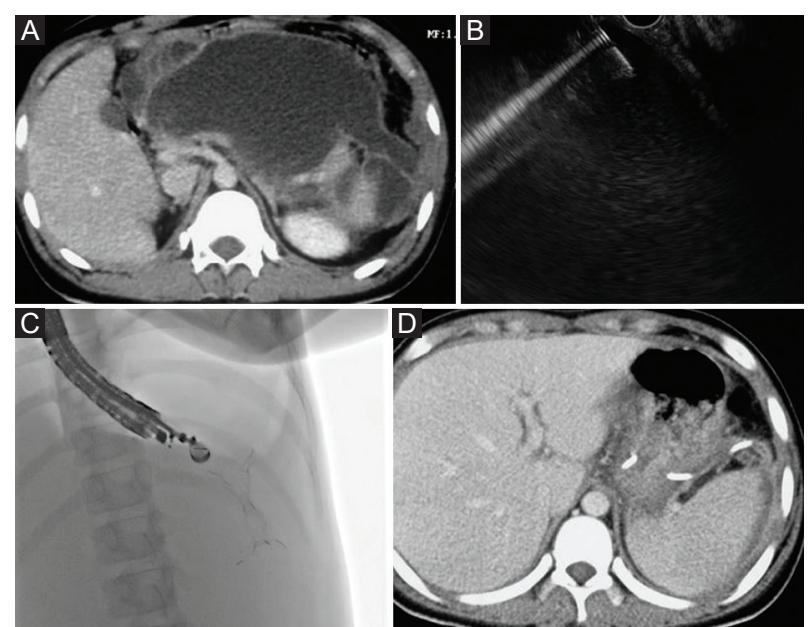

Figure 1 (A) Computed tomography (CT) of the abdomen: Large post-traumatic collection with an incompletely formed wall. (B) Endoscopic ultrasound (EUS)-guided drainage of fluid collection. (C) EUS-guided lumen apposing metal stent (LAMS) placed to drain pancreatic fluid collection (PFC). (D) CT abdomen: resolved PFC with LAMS replaced with a permanent indwelling transmural stent

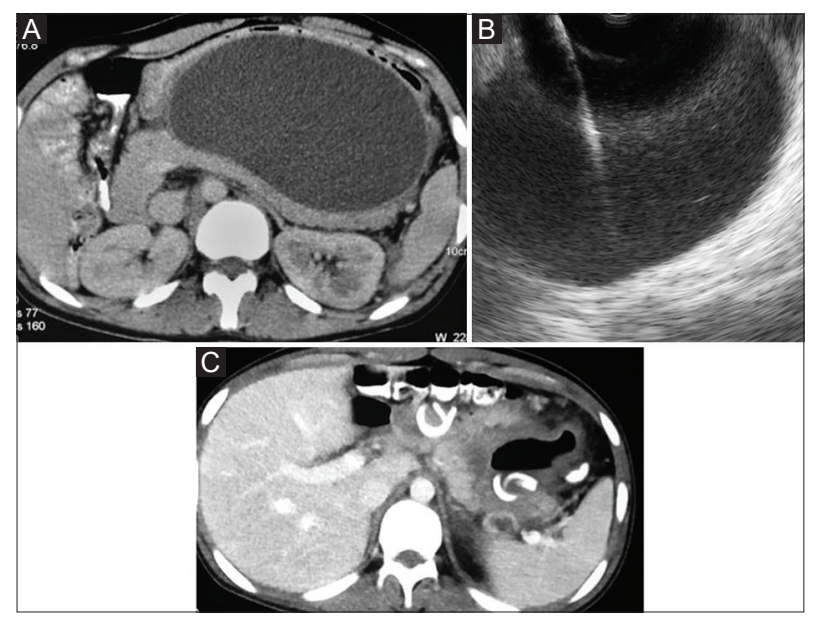

Figure 2 (A) Computed tomography (CT) of the abdomen: Large post-traumatic collection with a well-formed wall. (B) Endoscopic ultrasound-guided drainage of fluid collection. A plastic stent was placed. (C) CT abdomen: resolved pancreatic fluid collection with a permanent indwelling transmural stent

causing acute pancreatitis and formation of PFC or pancreatic fistulas [1]. As it is minimally invasive, safe and effective, EUSguided transmural drainage is the preferred treatment option for pancreatic pseudocysts and walled off necrosis [21]. Despite an abundance of data on the endoscopic drainage of pseudocysts, the experience with endoscopic drainage of post-traumatic PFC is limited [1,4]. Post-traumatic PFC and fistulas with partial pancreatic duct disruption have been successfully treated with bridging transpapillary stent [1-9,11,17,18]. However, endoscopic transpapillary drainage is usually ineffective in patients with complete MPD disruption and these patients usually require surgical intervention for a successful outcome. Few patients with complete MPD disruption and a bulging PFC into the stomach or duodenum have been treated with endoscopic 
transmural drainage $[1,4]$. The advent of EUS has revolutionized the management of PFC with even non-bulging, and distant PFC being successfully treated endoscopically $[22,23]$. However, experience with EUS-guided transmural drainage of posttraumatic PFC is limited $[1,4,18]$. Also, the appropriate timing of EUS-guided transmural drainage is unclear.

The management of pancreatic traumatic injury in the early phase involves supportive care with attempts to accurately diagnose the MPD injury as the definitive management depends on integrity of the MPD along with the extent of pancreatic parenchymal damage [1,4]. The American Association for the Surgery of Trauma Organ Injury Scaling Committee has described a grading system that can help in the decision of the appropriate management [24]. Patients with grade I and II lesions have no ductal injury and these patients can be successfully managed with conservative management. On the other hand, patients with grade $\mathrm{V}$ injury have massive disruption of the head of pancreas and usually need surgery. Patients with grade III and IV lesions have distal or proximal transection of the pancreas respectively and the traditional management of these patients involved either surgical resection or drainage depending on the extent of the ductal and parenchymal injury. A selected group of hemodynamically stable patients with isolated MPD injury with partial duct disruption have been successfully treated with endoscopic transpapillary drainage in the early phase of the illness $[1,7,8]$. However, transpapillary drainage alone is associated with infections especially in patients with large $(>6 \mathrm{~cm})$ PFC and is usually infective in complete MPD disruption [13]. The ductal anatomy and disruption can be demonstrated on magnetic resonance cholangiopancreatography and in the current era there is no role for diagnostic ERCP. In the current study, we have demonstrated that EUS-guided transmural drainage is a safe and effective minimally invasive therapeutic option in this group of patients.

EUS-guided transmural drainage of PFC is usually applied in collections that have a well-formed enclosing wall and this usually occurs after 4 weeks from the onset of illness [14]. Early ( $<4$ weeks) endoscopic transmural drainage of PFC is not advocated due to the fear of an increased risk of complications in patients with incompletely formed enclosing wall. However, few recent studies in patients with pancreatic necrotic collections have reported that early ( $<4$ weeks) endoscopic transmural drainage is safe and effective $[25,26]$. We also found that early EUS-guided transmural drainage of post-traumatic PFC is safe and effective. In our study, 3 patients had an incompletely formed wall and endoscopic drainage was done earlier than 4 weeks in $8(61 \%)$ patients. Also, $84 \%$ patients had complete MPD disruption and leaving transmural stents in situ in these patients for an indefinite period probably keeps the iatrogenic transmural tract patent and thus reduces the risk of recurrence of PFC due to DPD syndrome [16]. None of our patients with complete duct disruption and indwelling transmural stent had either recurrence of PFC or abdominal pain.

The issue of antibiotic prophylaxis while draining sterile PFCs is a debatable issue. Since endoscopic manipulation of these sterile cysts drains them into the contaminated gastrointestinal tract, prophylactic administration of post-procedural antibiotics is advisable, although the data supporting this practice is scanty. The American Society of Gastrointestinal Endoscopy guidelines recommend prophylactic antibiotic use for EUSguided sampling of pancreatic cystic lesions [27]. However, recent evidence suggests that antibiotic prophylaxis may not be needed after EUS fine needle aspiration of cysts, especially once the cysts have been completely aspirated [28]. However, large sized PFC take some time for significant resolution even after adequate large diameter drainage and therefore we follow the practice of giving antibiotics for 5-7 days.

Our study is associated with a few limitations. Firstly, it is a retrospective study from a tertiary teaching hospital and thus has an inherent drawback of referral bias. It is a single-center study with a small sample size and variable interventions

Table 1 Clinical and demographic profile of patients with post-traumatic pancreatic fluid collections (PFC)

\begin{tabular}{|c|c|c|c|c|c|c|c|}
\hline No. & Age/Sex & Symptoms & Size of PFC $(\mathrm{cm})$ & $\begin{array}{l}\text { Pancreatic duct } \\
\text { disruption }\end{array}$ & $\begin{array}{l}\text { Transmural stent } \\
\text { placed }\end{array}$ & $\begin{array}{c}\text { Period of } \\
\text { resolution (weeks) }\end{array}$ & $\begin{array}{l}\text { Follow up } \\
\text { (months) }\end{array}$ \\
\hline 1 & $22 / \mathrm{M}$ & Abdominal pain & 6 & Complete & Plastic & 3 & 7 \\
\hline 2 & $34 / \mathrm{M}$ & Abdominal pain & 16 & Partial & LAMS & 3 & 21 \\
\hline 3 & $18 / \mathrm{M}$ & Abdominal pain & 12 & Complete & Plastic & 3 & 73 \\
\hline 4 & $18 / \mathrm{M}$ & Abdominal pain & 8 & Complete & Plastic & 2 & 21 \\
\hline 5 & $19 / \mathrm{M}$ & Abdominal pain & 10 & Complete & Plastic & 3 & 58 \\
\hline 6 & $18 / \mathrm{M}$ & Abdominal pain & 14 & Complete & Plastic & 2 & 26 \\
\hline 7 & $18 / \mathrm{M}$ & Abdominal pain & 15 & Complete & Plastic & 3 & 48 \\
\hline 8 & $23 / \mathrm{M}$ & Abdominal pain & 12 & Complete & Plastic & 3 & 32 \\
\hline 9 & 19/M & Abdominal pain & 10 & Complete & Plastic & 3 & 24 \\
\hline 10 & $21 / \mathrm{M}$ & Abdominal pain & 17 & Partial & LAMS & 3 & 14 \\
\hline 11 & $28 / \mathrm{M}$ & Abdominal pain & 14 & Complete & Plastic & 3 & 9 \\
\hline 12 & $21 / \mathrm{F}$ & Abdominal pain & 12 & Complete & Plastic & 3 & 6 \\
\hline 13 & $21 / \mathrm{M}$ & Abdominal pain & 8 & Complete & Plastic & 2 & 5 \\
\hline
\end{tabular}

LAMS, lumen apposing metal stent 
have been performed in the included patients. Moreover, the endoscopic procedures were conducted in a unit with extensive experience in interventional EUS and pancreatic endotherapy and therefore the results may not be generalizable.

\section{Conclusion}

In conclusion, EUS-guided ransmural drainage of posttraumatic PFC is safe and effective. Moreover, it can be safely performed in patients with symptomatic PFC in the early phase ( $<4$ weeks) after pancreatic trauma.

\section{Summary Box}

\section{What is already known:}

- Patients with post-traumatic pancreatic fluid collections (PFC) have been conventionally treated with either percutaneous drainage or surgery

- The role of endotherapy is limited to patients with partial pancreatic duct disruptions and small PFC

- Endoscopic ultrasound (EUS)-guided transmural drainage is a safe and effective minimally invasive treatment option for pseudocysts and walled off necrosis

\section{What the new findings are:}

- EUS-guided transmural drainage of post-traumatic PFC is safe and effective

- EUS-guided transmural drainage of post-traumatic PFC can be safely performed in the early phase $(<4$ weeks) after pancreatic trauma

\section{References}

1. Bhasin DK, Rana SS, Rawal P. Endoscopic retrograde pancreatography in pancreatic trauma: need to break the mental barrier. J Gastroenterol Hepatol 2009;24:720-728.

2. Wind P, Tiret E, Cunningham C, et al. Contribution of endoscopic retrograde pancreatography in management of complications following distal pancreatic trauma. Am Surg 1999;65:777-783.

3. Kim S, Kim JW, Jung PY, et al. Diagnostic and therapeutic role of endoscopic retrograde pancreatography in the management of traumatic pancreatic duct injury patients: Single center experience for 34 years. Int J Surg 2017;42:152-157.

4. Bhasin DK, Rana SS, Rao C, et al. Endoscopic management of pancreatic injury due to abdominal trauma. Jop 2012;13:187-192.

5. Lin BC, Liu NJ, Fang JF, et al. Long-term results of endoscopic stent in the management of blunt major pancreatic duct injury. Surg Endosc 2006;20:1551-1555.

6. Lin BC, Wong YC, Chen RJ, et al. Major pancreatic duct continuity is the crucial determinant in the management of blunt pancreatic injury: a pancreatographic classification. Surg Endosc 2017;31:4201-4210.

7. Thomson DA, Krige JE, Thomson SR, et al. The role of endoscopic retrograde pancreatography in pancreatic trauma: a critical appraisal of 48 patients treated at a tertiary institution. J Trauma Acute Care Surg 2014;76:1362-1366.

8. Kim HS, Lee DK, Kim IW, et al. The role of endoscopic retrograde pancreatography in the treatment of traumatic pancreatic duct injury. Gastrointest Endosc 2001;54:49-55.

9. Houben $\mathrm{CH}$, Ade-Ajayi N, Patel S, et al. Traumatic pancreatic duct injury in children: minimally invasive approach to management. $J$ Pediatr Surg 2007;42:629-635.

10. Subramanian A, Dente CJ, Feliciano DV. The management of pancreatic trauma in the modern era. Surg Clin North Am 2007;87:1515-1532, x.

11. Jurkovich GJ, Carrico CJ. Pancreatic trauma. Surg Clin North Am 1990; 70:575-93.

12. Patton JH, Jr., Fabian TC. Complex pancreatic injuries. Surg Clin North Am 1996;76:783-795.

13. Varadarajulu S, Rana SS, Bhasin DK. Endoscopic therapy for pancreatic duct leaks and disruptions. Gastrointest Endosc Clin N Am 2013;23:863-892.

14. Rana SS. An overview of walled-off pancreatic necrosis for clinicians. Expert Rev Gastroenterol Hepatol 2019;13:331-343.

15. Singhal S, Rotman SR, Gaidhane $M$, et al. Pancreatic fluid collection drainage by endoscopic ultrasound: an update. Clin Endosc 2013;46:506-514.

16. Verma S, Rana SS. Disconnected pancreatic duct syndrome: updated review on clinical implications and management. Pancreatology 2020;20:1035-1044.

17. Lin BC, Fang JF, Wong YC, et al. Blunt pancreatic trauma and pseudocyst: management of major pancreatic duct injury. Injury 2007;38:588-593.

18. Grossjohann HS, Kristensen TS, Hansen CP. Endoscopic ultrasound-guided drainage of a pancreatic pseudocyst after a bicycle trauma. Ultrasound Int Open 2019;5:E75-E77.

19. Rana SS, Sharma V, Sharma R, et al. Endoscopic ultrasound guided transmural drainage of walled off pancreatic necrosis using a "step - up" approach: a single centre experience. Pancreatology 2017;17:203-208.

20. Cotton PB, Eisen GM, Aabakken L, et al. A lexicon for endoscopic adverse events: report of an ASGE workshop. Gastrointest Endosc 2010;71:446-454.

21. Guo J, Saftoiu A, Vilmann P, et al. A multi-institutional consensus on how to perform endoscopic ultrasound-guided peri-pancreatic fluid collection drainage and endoscopic necrosectomy. Endosc Ultrasound 2017;6:285-291.

22. Rana SS, Bhasin DK. Nonfluoroscopic endoscopic ultrasoundguided transmural drainage of pseudocysts: A pictorial technical review. Endosc Ultrasound 2015;4:92-97.

23. Rana SS, Dawra S, Sharma R, et al. Clinical manifestations, imaging features, and endoscopic management of renal pseudocysts: a case series. Ann Gastroenterol 2020;33:313-317.

24. Moore EE, Cogbill TH, Malangoni MA, et al. Organ injury scaling, II: Pancreas, duodenum, small bowel, colon, and rectum. J Trauma 1990;30:1427-1429.

25. Trikudanathan G, Tawfik P, Amateau SK, et al. Early ( $<4$ weeks) versus standard ( $\geq 4$ weeks) endoscopically centered step-up interventions for necrotizing pancreatitis. Am J Gastroenterol 2018;113:1550-1558.

26. Patel R, Gardner TB. Early versus late necrotic pancreatic fluid collection drainage: Does timing make a difference? Gastrointest Endosc 2020;91:1310-1311.

27. Muthusamy VR, Chandrasekhara V, Acosta RD, et al. The role of endoscopy in the diagnosis and treatment of cystic pancreatic neoplasms. Gastrointest Endosc 2016;84:1-9.

28. Hashimoto R, Lee JG. Concise commentary: antibiotic prophylaxis for endoscopic needle aspiration of pancreatic cystic lesions: bursting the bubble? Dig Dis Sci 2019;64:2316-2317. 